\title{
Bacteriological Profile of Burn Patients in a Tertiary Health Care Centre
}

\author{
Ish Garg ${ }^{1}$, Pinki Pargal², Aroma Oberoi ${ }^{3}$, Vijay A. E. Obed ${ }^{4}$ \\ ${ }^{1}$ Department of Plastic Surgery, Christian Medical College, Ludhiana, Punjab, India. ${ }^{2}$ Department of Plastic Surgery, \\ Christian Medical College, Ludhiana, Punjab, India. ${ }^{3}$ Department of Microbiology, Christian Medical College, Ludhiana, \\ Punjab, India. ${ }^{4}$ Department of Plastic Surgery, Christian Medical College, Ludhiana, Punjab, India.
}

\section{ABSTRACT}

\section{BACKGROUND}

burn injury is the major public health problem in many parts of the world. It has been estimated that $75 \%$ of all deaths following burns are related to infections. Burn wound sepsis leads to both local and systemic manifestations as the burn injury destroys the skin barrier that normally protects \& prevents the invasion by microorganisms, making the burn wound exposed to various infections. Topical antimicrobial decreases the microbial overgrowth but rarely prevents further colonization with other potentially invasive bacteria \& fungi. Organisms keep on changing over the time. So, in depth knowledge of the organisms that are predominant in that particular period along with their sensitivity pattern is vital as many septic burn patients need to be treated with antibiotics much before the results of wound culture sensitivity are available. This would be crucial to reduce overall infection related morbidity \& mortality.

\section{METHODS}

A prospective study was done in burn patients admitted in burn unit of Christian Medical College and Hospital, Ludhiana, during the period 2012-2013. A total of 103 patients was studied after following inclusion and exclusion criteria. Wound swab (surface smears) and biopsy/tissue culture were done on first-, second-, third- and fourth-week post admission. Organisms prevalent and antibiotic sensitivity at that particular period were determined. All types of burn patients were included from OPD and emergency department after an informed consent was obtained. All those who were not willing for participation in the study were excluded.

\section{RESULTS}

The most common mode of injury in our study was flame burn. Pseudomonas \& Acinetobacter were most common microorganisms causing wound infections after one week of hospital stay. After that MRSA was the dominant organism.

\section{CONCLUSIONS}

Identification of microorganisms causing burn wound infections by wound swab cultures/tissue cultures and administering antibiotics according to their antibiotic sensitivity patterns is pivotal in managing these infections and decreasing overall morbidity and mortality in burns unit.

\section{KEY WORDS}

Burns, Wound Swab, Tissue Culture, Microorganisms, Antibiotic Sensitivity
Corresponding Author:

Dr. Pinki Pargal,

Associate Professor,

Department of Plastic Surgery,

Christian Medial College,

Ludhiana, Punjab, India.

E-mail: pinkipargal@gmail.com

DOI: $10.14260 / \mathrm{jemds} / 2019 / 814$

Financial or Other Competing Interests: None.

How to Cite This Article:

Garg I, Pargal P, Oberoi A. et al. Bacteriological profile of burn patients in a tertiary health care centre. J. Evolution Med. Dent. Sci. 2019;8(50):3759-3763, DOI: $10.14260 /$ jemds/2019/814

Submission 26-10-2019,

Peer Review 27-11-2019,

Acceptance 03-12-2019,

Published 16-12-2019. 


\section{BACKGROUND}

Burn injury is a major health problem in many parts of the world. It has been estimated that $75 \%$ of all deaths following burns are related to burn wound infection or other related infections \& complications. Burn injury destroys the skin barrier that normally prevents invasion by microorganisms. These makes the burn wound the most frequent origin of sepsis in these patients. Further burn injuries induce a state of immunosuppression that leads to infectious complications. (1) The several rates of burn patients have improved substantially in the past few decades due to advances in modern medical care in specialized burn centers which included advances in fluid resuscitation, nutritional support, burn wound care however, the advances in infection control practices that has markedly improved the outcome. Burn wound infections are one of the most important and potentially serious complications that occur in the acute period following injury. (2) The important factor that has played a significant role in decreasing the overall fatality rates from burn wound infection and sepsis include the use of topical and prophylactic antibiotics and advances in infection control measures on modern burn units. Most of our understanding of the epidemiology of burn wounds infections has been gleaned from studies carried out in the 1950's. through 1990 during the pre-excision era of burn care. (3) It is not surprising that the overall morbidity and mortality of burn wound infections, tissue invasion and secondary sepsis were extremely high during this time period because the growth of bacteria on the burn wound surface was controlled but not eradicated. Case fatality rates were $40 \%$ or higher depending on the extent of burn injury. (4) Immediate colonization by the patient's normal skin flora (i.e. Staphylococcus aureus and Streptococcus pyogenes) occurred following injury. (5) Subsequent colonization by the patient's own gut flora added to the complex microbial ecology on the burn wound surface shortly thereafter. (6) Nosocomial transmission of microorganism to the burn wound also occurred by transfer from the hands of health care personnel and through immersion hydrotherapy treatment. (7) burn unit outbreaks of infection were attributed mainly to contaminated hydrotherapy tanks or water but in other cases to contaminated surfaces such as the patient's mattress. (8) Thermal destruction of the skin barrier and concomitant depression of local and systemic host cellular and humoral immune responses are pivotal factors contributing to infectious complications in patients with severe burns. (9) The burn wound surface (In deep partial thickness and in full thickness burns) is a protein rich environment consisting of a vascular necrotic tissue (eschar) that provides a favourable niche for microbial colonization and proliferation. (10) The avascularity of the Eschar results in impaired migration of the Host immune cells and restricts delivery of systematically administered antimicrobial agents to the area, while toxic substances released by eschar tissue impair local immune responses. (10) Although burn wound surfaces are sterile, immediately following thermal injury. These wound eventually become colonized with microorganisms. (11) The nature and extent of the thermal injury along with the types and amounts of microorganisms colonizing the burn wound appear to influence the failure risk of an invasive wound infection. (10) Gram positive bacteria that survives the thermal insult, such as staphylococci located deep within sweat glands and hair follicles, heavily colonize the wound surface within the first 48 hours unless topical anti-microbial agents are used. (12) Eventually (after an average of 5-7 days) theses wounds are subsequently colonized with other microbes including gram negative bacteria and yeasts derived from the host's normal ggastrointestinaland upper respiratory flora and/or from the hospital environment or that are transferred by health care worker's hand. (12) If the patients (host) defence measures (including the excision of necrotic tissues and wound care) are inadequate or delayed microbial invasion of viable tissue occurs, which is the hallmark of an invasive burn wound infection. The present study was done to find out the spectrum of wound infection in burn patients and the antibiotic sensitive to the particular organism causing burn infection.

We wanted to study the pattern of colonization and antibiotic sensitivity pattern of infectious organisms in burn wound.

\section{METHODS}

A prospective study was done in burn patients admitted in burn unit of Christian medical college and hospital Ludhiana in a period between 2012-2013. Total number of 103 patients were studied after following inclusion and exclusion criteria Patients with flame burns, electric contact and flash burns, chemical burns and hot water/ liquid scald burns were included in the study. Patients not willing for participation in the study were excluded from the study.

\section{Wound Treatment}

Closed dressings using silver sulfadiazine ointment were used in most of the patients. The burn wounds were washed daily to remove necrotic tissue and the remnants of the previous day's ointment. In patients developing side effects like neutropenia, thrombocytopenia due to silver sulfadiazine and in patients with superficial burns, Neosporin ointment was used for dressing. In patients with MRSA infection, TBact ointment was used for dressing.

\section{Procedures Used for Wound Sampling}

1. Wound Swab (surface smears)- Microbial colonization of all wounds was studied from the time of admission to discharge. On admission, the sampling procedure included swabs that are taken from clinically deep areas of the burn wound prior to any cleansing. Swabs were taken on Day 1,7 , 14,21 . The bandages will be removed, the remnants of the previous day's ointment washed, and the wounds swabbed and cultured as follows: A sterile cotton swab moistened with sterile normal saline was rubbed onto the burn wound surface. Swabs were taken from the areas which appeared deep, areas with discharge, thick eschar, preferably away from urogenital region and other potentially contaminated sites. The swabs were then sent for culture.

2. Biopsy Culture- Biopsy sample from area of the burn wound was obtained and sent for microbiological workup of the pathogens and their resistance profiles. Biopsy cultures were taken on Day 1, 7, 14, 21. It was taken from the site of deep burn, preferably away from urogenital region and other 
potentially contaminated sites. After cleaning the wound with isopropyl alcohol, 2 parallel incisions $1-2 \mathrm{~cm}$ in length and 1.5 $\mathrm{cm}$ apart with a depth to obtain a portion of the underlying fat were made in the skin. Then, the sample was be preserved in normal saline solution and labelled. And send to Microbiology lab for further evaluation.

\section{Blood C/S}

Blood culture and sensitivity was sent in cases where systemic infection was suspected by presence of high spiking fever or other signs of septicaemia and noted.

\section{Microbiology}

The swabs/biopsy specimens were transported to the laboratory for processing immediately as per protocols for aerobic, anaerobic and fungal cultures. They were streaked onto a differential medium [e.g. MacConkey agar] and an enriched medium (e.g. blood agar). These plates were incubated at $37^{0} \mathrm{C}$ for 24 hours. The organisms isolated from various wound swab/ tissue biopsy cultures were noted along with their colony count. Antibiotic sensitivity of isolates obtained from the burn wound were carried out by Kirby Bauer method as per protocols and noted.

\section{Statistical Analysis}

Basic patient data like age, sex, mode of injury, percentage of burns, type of burns, depth of burns, associated comorbidities, routine investigations, whether patient underwent surgery or not, outcome and local/systemic antibiotics used were noted. Proportions and chi square test were used to analyse the data collected.

\section{RESULTS}

\begin{tabular}{|c|c|c|}
\hline Local Antibiotic Used & No. of Patients & Percent \\
\hline Silver Sulfadiazine & 77 & 74.8 \\
\hline Neosporin & 26 & 25.2 \\
\hline T-Bact & 6 & 5.8 \\
\hline \multicolumn{2}{|c|}{ Table 1. Local Antibiotic Used } \\
\hline$>$ 1 type of ointment was used in many patients
\end{tabular}

\begin{tabular}{|c|c|c|}
\hline Systemic Antibiotic Used & Total No. of Patients & Percent \\
\hline Ampicillin & 75 & 72.8 \\
\hline Amikacin & 86 & 83.5 \\
\hline Cefazolin & 21 & 20.4 \\
\hline Netromycin & 1 & 1.0 \\
\hline Ofloxacin & 1 & 1.0 \\
\hline Ciprofloxacin & 3 & 2.9 \\
\hline Polymyxin B & 11 & 10.7 \\
\hline Imipenem & 20 & 19.4 \\
\hline Piperacillin/Tazobactam & 5 & 4.9 \\
\hline Cefoperazone/Sulbactam & 6 & 5.8 \\
\hline Teicoplanin & 8 & 7.8 \\
\hline Linezolid & 18 & 17.5 \\
\hline \multicolumn{2}{|c|}{ Table 2. Systemic Antibiotic Used } \\
\hline \multicolumn{2}{|c|}{} \\
\hline$>1$ type of systemic antibiotic was used in most of the patients. \\
\hline
\end{tabular}

\begin{tabular}{|c|c|c|c|c|}
\hline Organisms & I & II & III & IV \\
\hline No Growth & 67 & 24 & 9 & 4 \\
\hline Acinetobacter & 7 & 18 & 16 & 17 \\
\hline E. coli & 8 & 7 & 4 & 3 \\
\hline Enterobacter & 4 & 4 & 3 & 2 \\
\hline Klebsiella & 3 & 5 & 7 & 4 \\
\hline Pseudomonas & 13 & 20 & 23 & 21 \\
\hline Citrobacter & 0 & 0 & 1 & 1 \\
\hline Proteus sp. & 0 & 2 & 0 & 1 \\
\hline MSSA & 8 & 4 & 3 & 1 \\
\hline MRSA & 5 & 13 & 9 & 7 \\
\hline Enterococcus & 6 & 2 & 1 & 1 \\
\hline
\end{tabular}

\begin{tabular}{|c|c|c|c|c|}
\hline Candida albicans & 0 & 1 & 0 & 0 \\
\hline Candida non - albicans & 0 & 1 & 3 & 0 \\
\hline Aspergillus & 0 & 0 & 1 & 0 \\
\hline Table 3. Organisms Grown in Wound Swab Cultures \\
\hline \\
>1 type of organism growth was seen in many patients \\
\hline
\end{tabular}

\begin{tabular}{|c|c|c|c|c|}
\hline Organisms & I & II & III & IV \\
\hline No Growth & 62 & 22 & 9 & 6 \\
\hline Acinetobacter & 9 & 19 & 15 & 13 \\
\hline E. coli & 9 & 9 & 8 & 4 \\
\hline Enterobacter & 3 & 2 & 2 & 2 \\
\hline Klebsiella & 2 & 4 & 3 & 5 \\
\hline Pseudomonas & 14 & 24 & 27 & 18 \\
\hline Citrobacter & 1 & 0 & 0 & 1 \\
\hline Proteus sp. & 1 & 3 & 1 & 1 \\
\hline MSSA & 4 & 3 & 4 & 1 \\
\hline MRSA & 7 & 10 & 7 & 5 \\
\hline Enterococcus & 6 & 8 & 4 & 1 \\
\hline Candida albicans & 0 & 1 & 0 & 0 \\
\hline Candida non - albicans & 0 & 0 & 0 & 0 \\
\hline Aspergillus & 1 & 0 & 0 & 0 \\
\hline \multicolumn{7}{|l|}{ Table 4. Organisms Grown in Tissue Cultures } \\
\hline$>1$ type of organism growth was seen in many patients. \\
\hline
\end{tabular}

\begin{tabular}{|c|c|c|c|c|c|c|c|c|}
\hline & \multicolumn{4}{|c|}{ Wound Swab } & \multicolumn{4}{|c|}{ Tissue } \\
\hline Antibiotic & I & II & III & IV & I & II & III & IV \\
\hline Chloramphenicol & 14 & 18 & 18 & 16 & 19 & 17 & 16 & 15 \\
\hline Cotrimoxazole & 11 & 10 & 8 & 9 & 17 & 9 & 6 & 9 \\
\hline Ampicillin & 3 & 0 & 2 & 0 & 7 & 4 & 4 & 0 \\
\hline Gentamycin & 11 & 7 & 7 & 2 & 21 & 13 & 8 & 2 \\
\hline Amikacin & 11 & 33 & 32 & 22 & 25 & 37 & 33 & 20 \\
\hline Netromycin & 14 & 22 & 22 & 12 & 22 & 23 & 19 & 11 \\
\hline Cefotaxime & 10 & 13 & 8 & 11 & 16 & 16 & 11 & 7 \\
\hline Cefoperazone & 11 & 9 & 4 & 7 & 15 & 11 & 8 & 4 \\
\hline Ceftazidime & 7 & 9 & 17 & 8 & 12 & 12 & 8 & 4 \\
\hline Ofloxacin & 18 & 14 & 15 & 7 & 18 & 23 & 10 & 7 \\
\hline Ciprofloxacin & 13 & 13 & 13 & 13 & 22 & 15 & 14 & 13 \\
\hline Cefepime & 1 & 3 & 8 & 5 & 4 & 5 & 7 & 3 \\
\hline Aztreonam & 2 & 1 & 3 & 7 & 1 & 2 & 4 & 5 \\
\hline Polymyxin B & 29 & 49 & 52 & 38 & 36 & 58 & 54 & 36 \\
\hline Colistin & 11 & 19 & 21 & 21 & 17 & 19 & 21 & 2 \\
\hline Imipenem & 27 & 44 & 41 & 34 & 34 & 50 & 39 & 30 \\
\hline Meropenem & 12 & 26 & 17 & 18 & 21 & 25 & 19 & 19 \\
\hline Ceftriaxone & 9 & 9 & 5 & 6 & 16 & 11 & 8 & 5 \\
\hline Tobramycin & 11 & 21 & 17 & 11 & 21 & 24 & 17 & 10 \\
\hline Piperacillin/Tazobactam & 19 & 22 & 20 & 21 & 22 & 28 & 19 & 20 \\
\hline Cefoperazone/Sulbactam & 16 & 19 & 16 & 16 & 24 & 26 & 19 & 13 \\
\hline Ticarcillin/Clavulanic acid & 0 & 3 & 2 & 3 & 6 & 5 & 2 & 3 \\
\hline Augmentin & 5 & 4 & 2 & 3 & 4 & 2 & 3 & 3 \\
\hline Vancomycin & 20 & 19 & 12 & 9 & 17 & 21 & 14 & 7 \\
\hline Teicoplanin & 18 & 18 & 13 & 8 & 17 & 20 & 15 & 6 \\
\hline Linezolid & 19 & 19 & 13 & 9 & 17 & 21 & 14 & 7 \\
\hline Clindamycin & 11 & 11 & 8 & 2 & 8 & 8 & 6 & 2 \\
\hline Erythromycin & 4 & 4 & 2 & 1 & 4 & 2 & 2 & 1 \\
\hline Moxifloxacin & 3 & 3 & 2 & 1 & 1 & 3 & 2 & 1 \\
\hline Tigecycline & 0 & 0 & 1 & 0 & 0 & 0 & 0 & 0 \\
\hline Azithromycin & 4 & 2 & 1 & 4 & 7 & 4 & 1 & 2 \\
\hline Itraconazole & 0 & 2 & 3 & 0 & 0 & 0 & 0 & 0 \\
\hline Fluconazole & 0 & 2 & 3 & 0 & 0 & 0 & 0 & 0 \\
\hline Clotrimazole & 0 & 2 & 3 & 0 & 0 & 0 & 0 & 0 \\
\hline Ketoconazole & 0 & 2 & 3 & 0 & 0 & 0 & 0 & 0 \\
\hline & ble & $\ln$ & $t$ & & & & & \\
\hline
\end{tabular}

\section{DISCUSSION}

Local antibiotic used were silver sulfadiazine ointment in $74.8 \%$. Neosporin ointment was used in $2.5 \%$ patients with first degree and superficial second-degree burns. T-bact ointment was used in $5.8 \%$ patients, especially in the patients with MRSA positive wound swab/tissue culture. More than one type of ointment was used in many patients.

In our study Amikacin and Ampicillin were the most commonly used systemic antibiotics in majority of patients (83.5\% \& 72.8\% respectively). Cefazolin was used in $20.4 \%$ of the patients. Later in the course of treatment, in patients with Resident Gram-negative bacilli wound infections; higher antibiotics like Imipenem (19.4\%), Polymyxin B (10.7\%) 
were given. In patient with MRSA positive cultures, linezolid $(17.5 \%)$ and teicoplanin (7.8\%) were given. Other antibiotic used were Cefoperazone/Sulbactam (5.8\%), Piperacillin/ Tazobactam (4.9\%), Ciprofloxacin (2.9\%), ofloxacin, Netromycin \& Erythromycin (1\% each). More than one type of systemic antibiotic was used in most of the patients.

In our study, wound swab culture was showed no growth in majority $(55.4 \%)$ of the patients. In rest of the patients most common organisms isolated were Gram negative bacilli (Pseudomonas, E. coli, Acinetobacter, Klebsiella, Enterobacter, Proteus, Citrobacter followed by Gram positive Cocci (MSSA, MRSA, Enterococcus) and even less commonly fungi (Candida albicans, Candida Non albicans, Aspergillus). More than one type of microorganism growth was seen in many patients. After hospitalization incidence of 'No Growth' in wound swab cultures gradually reduced. Incidence of infection gradually increased; Pseudomonas and Acinetobacter was the most common organism to cause wound infections after $1^{\text {st }}$ week of hospital stay.

Similar results were obtained by Agnihotri et al (2004) in a five year study of burn unit where Pseudomonas Aeruginosa was found to be most common isolate in wound cultures followed by Staphylococcus aureus and Acinetobacter. (13) In study by Altoparlak U et al (2004) in burn patients Coagulase negative staphylococci \& Staphylococcus aureus were the most prevalent isolates in admission cultures; these were superseded by Pseudomonas aeruginosa in second week. (14) Setal (2004) in their study found staph aureus most prevalent isolate in admission with marked increase in number of staph aureus \& Pseudomonas from admission to $21^{\text {st }}$ day. (15) In contrast to our study there was a rise in the isolation rate of Acinetobacter species (86.3\%) after first week of admission. In burn patients in study done by Keen et al (2010). (16)

On admission tissue culture showed no growth (51.2\%)of the patients like wound swab cultures, most common organisms isolated were gram negative bacilli (Pseudomonas), E. coli, Acinetobacter, Klebsiella, Enterobacter, Proteus, Citrobacter) followed by Gram positive cocci (MSSA, MRSA, Enterococcus) and fungi (Candida albicans, Candida non albicans, Aspergillus). More than one type of microorganism growth was seen in many patients, after hospitalization, incidence of 'No growth' in tissue cultures gradually reduced. Incidence of infection gradually increased, Pseudomonas \& Acinetobacter being the most common organism to cause wound infections after one week of Hospital stay.

In our study Gram negative bacilli (Pseudomonas, Acinetobacter, E. coli) were resistant to common antibiotics like ampicillin, Cefotaxime, cotrimoxazole, ciprofloxacin in majority of the cases. The organisms were sensitive to Amikacin, Cefoperazone/sulbactam, Piperacillin/Tazobactam, Imipenem \& Polymyxin B. Gram positive cocci (MSSA, MRSA) were mostly sensitive to Teicoplanin, Linezolid, vancomycin and erythromycin. Fungi (Candida, Aspergillus) were sensitive to common anti fungals like clotrimazole, fluconazole, itracomazole \& ketoconazole. Similar results were seen by Agnihotri et al (2004) where amikacin was found to be the most effective doing against GM Negative Bacteria. In Gregory M et al (2013) staphylococcus spp. Strains isolated from patients' samples were sensitive to linezolid. (17)

\section{CONCLUSIONS}

Amikacin and Ampicillin were the most commonly used systemic antibiotics. On admission, wound swab and tissue cultures showed no growth in majority of the patients. Pseudomonas and Acinetobacter are the most common microorganisms causing wound infections after one week of hospital stay. These are resistant to common antibiotics and are sensitive to higher antibiotics in majority of the cases. Prevalence of MRSA is increasing and needs to be managed with appropriate antibiotics. Thus, diagnosis of microorganisms causing burn wound infections by wound swab cultures/tissue cultures and administering antibiotics according to their antibiotic sensitivity patterns is pivotal in managing these infections and decreasing overall morbidity and mortality in burns unit.

\section{REFERENCES}

[1] Atiyeh BS, Gunn SW, Hayek SN. State of the art in burn treatment. World J Surg 2005;29 (2):131-48.

[2] Appelgren P, Bjornhagen V, Bragderyd K, et al. A prospective study of infections in burn patients. Burns 2002;28 (1):39-46.

[3] Mayhall CG. The epidemiology of burn wound infections: then and now. Clin Infect Dis 2003;37 (4):543-50.

[4] Mason AD, McManus AT, Pruitt BA Jr. Association of burn mortality and bacteremia. A 25-year review. Arch Surg 1986;121 (9):1027-31.

[5] Bang RL, Gang RK, Sanyal SC, et al. Beta-haemolytic Streptococcus infection in burns. Burns 1999;25 (3):2426.

[6] Deutsch DH, Miller SF, Finley RK Jr. The use of intestinal antibiotics to delay or prevent infections in patients with burns. J burn Care Rehabil 1990;11 (5):436-42.

[7] Cardany CR, Rodeheaver GT, Horowitz JH, et al. Influence of hydrotherapy and antiseptic agents on burn wound bacterial contamination. J burn Care Rehabil 1985;6 (3):230-2.

[8] Embil JM, McLeod JA, Al-Barrak AM, et al. An outbreak of methicillin resistant Staphylococcus aureus on a burn unit: potential role of contaminated hydrotherapy equipment. Burns 2001;27 (7):681-8.

[9] Alexander JW. Mechanism of immunologic suppression in burn injury. J Trauma 1990;30 (Suppl 12):S70-5.

[10] Barret JP, Herndon DN. Effects of burn wound excision on bacterial colonization and invasion. Plast Reconstr Surg 2003;111 (2):744-50.

[11] Erol S, Altoparlak U, Akcay MN, et al. Changes of microbial flora and wound colonization in burned patients. Burns 2004;30 (4):357-61.

[12] Altoparlak U, Erol S, Akcay MN, et al. The time-related changes of antimicrobial resistance patterns and predominant bacterial profiles of burn wounds and body flora of burned patients. Burns 2004;30 (7):660-4.

[13] Agnihotri N, Gupta V, Joshi RM. Aerobic bacterial isolates from burn wound infections and their antibiograms - a five-year study. Burns 2004;30 (3):241-3. 
[14] Altoparlak U, Erol S, Akcay MN, et al. The time-related changes of antimicrobial resistance patterns and predominant bacterial profiles of burn wounds and body flora of burned patients. Burns 2004;30 (7):660-4.
[15] Keen EF 3rd, Robinson BJ, Hospenthal DR, et al. Incidence $\&$ bacteriology of burn infections at a military burn center. Burns 2010;36 (4):461-8.

[16] Anslead GM, Cadena J, HetaJaveri. Methods in Molecular biology. Vol. 1085. MIMN 2013. 